\title{
Epidural electrical stimulation of posterior structures of the human lumbosacral cord: 3 . Control of spasticity
}

\author{
MM Pinter ${ }^{1}$, F Gerstenbrand ${ }^{2}$ and MR Dimitrijevic*,2,3 \\ ${ }^{1}$ Maria Theresien Schloessel Neurological Hospital, Vienna, Austria; ${ }^{2}$ Ludwig Boltzmann Institute for Restorative \\ Neurology and Neuromodulation, Vienna, Austria; ${ }^{3}$ Department of Physical Medicine and Rehabilitation, Baylor \\ College of Medicine, Houston, Texas, USA
}

\begin{abstract}
Objectives: The purpose of this study was to evaluate the effect of spinal cord stimulation (SCS) on severe spasticity of the lower limbs in patients with traumatic spinal cord injury (SCI) under close scrutiny of the site and parameters of stimulation.

Materials and methods: Eight SCI patients (four women, four men) were included in the study. Levels of spasticity before and during stimulation were compared according to a clinical rating scale and by surface electrode polyelectromyography (pEMG) during passive flexion and extension of the knee, supplemented by a pendulum test with the stimulating device switched either on or off over an appropriate period.

Results: Both the clinical and the experimental parameters clearly demonstrated that SCS, when correctly handled, is a highly effective approach to controlling spasticity in spinal cord injury subjects. The success of this type of treatment hinges on four factors: (1) the epidural electrode must be located over the upper lumbar cord segment (L1, L2, L3); (2) the train frequency of stimulation must be in the range of $50-100 \mathrm{~Hz}$, the amplitude within $2-7 \mathrm{~V}$ and the stimulus width of $210 \mu \mathrm{s}$; (3) the stimulus parameters must be optimized by clinically assessing the effect of arbitrary combinations of the four contacts of the quadripolar electrode; and (4) amplitudes of stimulation must be adjusted to different body positions.

Conclusions: Severe muscle hypertonia affecting the lower extremities of patients with chronic spinal cord injuries can be effectively suppressed via stimulation of the upper lumbar cord segment.
\end{abstract}

Spinal Cord (2000) 38, 524-531

Keywords: spasticity; spinal cord stimulation; upper lumbar spinal cord segment

\section{Introduction}

In a pioneer study published in 1973 on the treatment of movement disorders, Cook and Weinstein described an effect of dorsal column stimulation on motor functions in multiple sclerosis patients, ${ }^{1}$ a finding that has since been confirmed by other investigators. ${ }^{2}$ The effectiveness of spinal cord stimulation (SCS) has also been demonstrated many times in other neurological conditions involved in head injuries, stroke, cerebral palsy, dystonia, spasmodic torticollis and degenerative diseases. $^{3-6}$

In 1979, Richardson et al succeeded in suppressing spasticity and flexor spasms in six patients with chronic spinal cord injury (SCI) involving clinically complete lesions of the thoracic cord by applying SCS with the electrode placed below the lesion. ${ }^{7}$ Siegfried et al., who treated 15 chronic SCI patients by placing the electrode above the lesion, failed to confirm this

*Correspondence: MR Dimitrijevic, Ludwig Boltzmann Institute for Restorative Neurology and Neuromodulation, Hofzeile 18-20, A-1190 Vienna, Austria finding. ${ }^{8}$ In an effort to clarify the precise impact of the site of stimulation, Dimitrijevic et al used SCS in a sample of 58 SCI patients and found spasticity to be more effectively controlled when the electrode was placed below, rather than above, the lesion. ${ }^{9}$ This finding was again confirmed by Barolat et al who, based on a total of 48 patients, concluded that SCS applied below the level of the lesion was an effective and safe approach to controlling SCI-related spasms. ${ }^{10}$

The 1990s saw the interest in this approach declining, mainly due to technical problems and the realization that SCS as a method to control spasticity was less effective in patients with severe spasms of the lower limbs. ${ }^{10,11}$ At the same time, however, SCS has established itself as a widely used method of controlling neurogenic pain, which is particularly effective if the induced paresthesia coincides with the area of pain distribution within the body. ${ }^{12}$

In our earlier work on the subject of motor control in human SCI we have described the complex neurocontrol mechanisms of the lumbar cord below 
the level of injury, illustrating the dependence of motor control of the injured spinal cord on residual supraspinal input. ${ }^{13-15}$ Furthermore, we have conducted a series of studies on the central pattern generator for locomotion in SCI patients and supplied evidence for stepping movement induced by SCS of posterior upper lumbar cord structures. ${ }^{16}$

Based on all these findings, we developed the hypothesis that an effect of SCS on lower limb spasticity can be exerted by activating the lumbar spinal cord network. These considerations prompted us to revisit the option of SCS in the management of severe spasticity caused by chronic SCI. We therefore performed the present study aimed at examining whether stimulation of the lumbar spinal cord can be optimized to effectively control severe spasticity of the lower limbs.

\section{Materials and methods}

\section{Patients}

Eight SCI patients (four women and four men) were included in the study. At the time of implanting the stimulation device, they were $28.1(18-34)$ years old, the post-injury interval averaged 41.5 (19-94) months. Seven injuries were due to car accidents, one was caused by a skiing accident (patient \#5). The study was approved by the local ethics committee, and all patients gave their informed consent. Pertinent demographic data are summarized in Table 1.

The following inclusion criteria were applied: (1) posttraumatic closed SCI in otherwise healthy adults; (2) injury longer than 1 year back; (3) severe spasticity as defined by an Ashworth score of $>2.0 ;^{17}$ (4) lack of response to antispastic medication; and (5) intact stretch and cutaneomuscular reflexes below the injury site. Excluded were SCI patients with ongoing infection or neurological complications of the primary disease.

Lesions were located at levels C5-6 $(n=3)$ and T3-6 $(n=5)$ and were categorized as A $(n=5), \mathrm{B}$ $(n=2)$ or $\mathrm{C}(n=1)$ according to the ASIA scale. ${ }^{18}$ ASIA motor scores ranged from 16 to 67 (mean
$41.38 \pm 15.03 \mathrm{SD})$, ASIA sensory scores ranged from 46 to 92 (mean 69.00 \pm 5.25 SD). Patients were clinically examined and classified according to Ashworth scores. ${ }^{17}$ Neurophysiological parameters included surface electrode EMG during relaxation, reinforcement maneuver and proprio-/exteroceptive reflexes. $^{19,20}$

All patients were on antispastic medication prior to and into the study until spasticity was effectively controlled by SCS. The regimens included baclofen at a mean daily dose of $62.5(25-100) \mathrm{mg}$ up to five times a day $(n=8)$, tizanidine $(n=3)$ and tetrazepam $(150 \mathrm{mg} /$ day; $n=1)$.

\section{Site of stimulation}

The surgical procedure of placing the electrode was carried out by a team comprised of a neurosurgeon or orthopedic surgeon, and a neurologist/clinical neurophysiologist. A quadripolar electrode (3487A, Medtronic) was placed transcutaneously in local anesthesia in the posterior epidural space at vertebral levels T11 to L1, and connected to an external stimulator (model 3625, Medtronic). The position of the electrode was verified by fluoroscopy (AP and lateral X-rays) and muscle twitch distribution patterns. ${ }^{21}$ Muscle twitches during intraoperative testing were elicited using a train of $5 \mathrm{~Hz}$ at a pulse width of $210 \mu \mathrm{s}$, intensifying the amplitude in $0.5-\mathrm{V}$ increments from 0 to 10 at two different polarities $(3-/ 0+$ versus $0-/ 3+)$. The definitive location of the electrode was determined based on muscle twitch responses by the quadriceps and adductor muscles elicited at an amplitude of $1-$ $4.5 \mathrm{~V}$ and a pulse width of $210 \mu \mathrm{s}$. For a detailed description of this procedure, the reader is referred to the first paper of this series. ${ }^{22}$ For neurosurgical intervention we followed the description given by Campus et al. ${ }^{23}$

\section{Laboratory and clinical assessment}

The effect of SCS on spasticity was verified by EMG recordings of passive stretch (hip-knee flexion/extension) manually performed for each lower limb. EMG

Table 1 Demographic data

\begin{tabular}{|c|c|c|c|c|c|c|c|c|c|c|c|c|}
\hline \multirow[b]{2}{*}{ \# } & \multirow[b]{2}{*}{ Sex } & \multirow{2}{*}{$\begin{array}{c}\text { Date of } \\
\text { birth }\end{array}$} & \multirow{2}{*}{$\begin{array}{l}\text { SCI } \\
\text { date }\end{array}$} & \multirow{2}{*}{$\begin{array}{l}\text { Level of } \\
\text { fracture }\end{array}$} & \multirow{2}{*}{$\begin{array}{c}\text { Frankel } \\
\text { grade }\end{array}$} & \multicolumn{2}{|c|}{ Level } & \multicolumn{2}{|c|}{$A S I A$} & \multicolumn{2}{|c|}{ Ashworth } & \multirow{2}{*}{$\begin{array}{l}\text { SCS } \\
\text { date }\end{array}$} \\
\hline & & & & & & mot. & sens. & mot. & sens. & $L L L$ & $R L L$ & \\
\hline 1 & $\mathrm{~m}$ & $21-04-70$ & $11-11-96$ & $\mathrm{C} 5 / 6$ & B & C6 & D6 & 21 & 74 & $3.0 / 3.0$ & $3.0 / 3.0$ & $13-04-99$ \\
\hline 2 & $\mathrm{~m}$ & $22-08-77$ & 24-06-94 & $\mathrm{C} 5 / 6$ & A & C6 & $\mathrm{C} 7$ & 16 & 46 & $2.5 / 2.5$ & $2.3 / 2.3$ & 26-07-99 \\
\hline 3 & $\mathrm{~m}$ & $22-02-70$ & 06-10-91 & $\mathrm{T} 4$ & A & D5 & D5 & 50 & 92 & $2.7 / 2.6$ & $1.2 / 1.2$ & $31-08-99$ \\
\hline 4 & $\mathrm{f}$ & $05-01-74$ & $05-11-94$ & $\mathrm{~T} 3 / 4$ & $\mathrm{C}$ & D6 & D6 & 67 & 87 & $3.2 / 3.2$ & $2.8 / 2.8$ & $01-12-97$ \\
\hline 5 & $\mathrm{~m}$ & 07-04-64 & $05-01-97$ & $\mathrm{C} 5 / 6$ & B & $\mathrm{C} 7$ & $\mathrm{C} 8$ & 27 & 74 & $3.2 / 3.2$ & $4.0 / 4.0$ & $05-08-98$ \\
\hline 6 & f & $07-09-66$ & $01-03-96$ & $\mathrm{~T} 4 / 5$ & A & D5 & D5 & 50 & 79 & $4.1 / 4.1$ & $3.5 / 3.5$ & $13-05-99$ \\
\hline 7 & $\mathrm{f}$ & $21-03-78$ & $25-12-94$ & $\mathrm{~T} 4 / 5$ & A & D5 & D5 & 50 & 50 & $3.5 / 3.5$ & $3.5 / 3.5$ & $04-10-96$ \\
\hline 8 & $\mathrm{f}$ & $12-02-65$ & 01-04-96 & $\mathrm{T} 5 / 6$ & A & D5 & D5 & 50 & 50 & $4.0 / 4.0$ & $4.0 / 4.0$ & $12-11-98$ \\
\hline
\end{tabular}

$\mathrm{SCI}=$ spinal cord injury; mot.= motor; sens.=sensor; $\mathrm{LLL}=$ left lower limb; RLL = right lower limb; SCS $=$ spinal cord stimulation 
activity was recorded with the patients in the supine position and the surface electrodes placed bilaterally over the quadriceps (Q), adductors (A), hamstring $(\mathrm{H})$, tibialis anterior (TA), triceps surae (TS), abdominal $(\mathrm{AB})$ and paraspinal $(\mathrm{P})$ muscles. Electrode impedance was maintained below $5 \mathrm{k} \Omega$. The EMGs were amplified using the Grass 12A5 system (Grass, Quincy, MA, USA) adjusted to a gain of 5000 over a bandwidth of $50-800 \mathrm{~Hz} \quad(-3 \mathrm{db})$ and digitized at 1800 samples/s/channel with a bit depth of 12 bits using the CODAS ADC system (DATQ Instruments, Akron, OH, USA). In addition to the EMG channels, two channels were allocated as level sensors or goniometer for the pendulum test documenting thigh angles, leg angles or foot position. The details of this procedure are described elsewhere. ${ }^{19,20}$ All recorded EMG data were stored and analyzed offline.

The effect of the epidural electrode was optimized by testing different combinations while the electrode was externalized. The four contacts $(0-3,0$ being the most rostral, 3 the most caudal) were used in pairs (cathode, anode) in 'bi-polar' stimulation mode with a stimulus rate of $50-100 \mathrm{~Hz}$ and an amplitude of $1-$ $10 \mathrm{~V}$.

In addition to the neurophysiological assessment, the effect on spasticity was independently evaluated by physiotherapists using a clinical rating scale based on the degree of resistance to passive stretch, frequency of spasms, the characteristics of irradiation pattern of the spasms and the persistence of such activity in lower limbs. ${ }^{11}$ According to this scale, the effect of SCS was considered (1) marked when spontaneous spasms and resistance to passive stretch were eliminated, (2) moderate when tonic spasticity was eliminated or reduced to residual phasic components, when spontaneous spasms were less frequent, shorter in duration and transmitted to fewer muscles; (3) marginal when spasticity was detectably reduced but involved no significant clinical benefit; and (4) absent when muscle hypertonia and frequency of spasms remained unchanged.

Whether or not spasticity was effectively controlled in the selected electrode position was decided based on clinical and neurophysiological findings including subjective parameters. In two patients, the temporary electrodes had to be repositioned to optimize the effect.

Once the beneficial effect of SCS was reproducibly established based on clinical and neurophysiological parameters over a 2-week period, the SCS electrode was internalized and connected to the implanted, programmable pulse generator (Itrel 3, Medtronic). This closed configuration allows subsequent switching from bipolar to unipolar stimulation mode by using one contact of the quadripolar electrode as cathode and the case of the implant as anode. SCS parameters were finally adjusted using the abovedescribed neurophysiological and clinical evaluation protocol.

\section{Data analysis}

The stimulation parameters used for the EMG recordings were identical to the ones the respective patients were using for continuous long-term SCS. Analog raw EMG data of three passive stretch maneuvers were rectified and root mean square (RMS) amplitudes of the manually selected portions computed using the Pegasus software. Data were normalized by calculating RMS amplitudes per second for all three passive stretch maneuvers. In a separate step, the RMS amplitudes of all maneuvers were aggregated as arithmetic means $( \pm S D)$ for the various muscles and for right versus left lower limbs (RLL, LLL). To analyze the effectiveness of SCS with respect to stimulation site, frequency and intensity, the mean RMS amplitudes and standard deviations of three passive stretch maneuvers at each sequence were calculated.

Pendulum test was also used to assess the muscle tone. $^{24}$ The RMS amplitude of the corresponding EMG (if present) was plotted against time in seconds. Then the total duration, the sweeps of the oscillations recorded with the goniometer and the degree of falling ankle of the first oscillation were calculated for both stimulation on and stimulation off.

\section{Statistical analysis}

Baseline RMS amplitudes ( $\mu \mathrm{V})$ during passive stretch obtained at study entry were compared to RMS amplitudes obtained under effective continuous SCS based on each separate muscle and on aggregated totals for all muscles. Student's $t$-test for independent samples was used to detect differences between preoperative and postoperative passive stretch reflex patterns. Wilcoxon's matched-pairs signed rank test was used to compare preoperative and postoperative (SCS-on) Ashworth scores. Student's $t$-test was also used to compare the effect on passive stretch at different stimulation sites, frequencies and intensities to preoperative baseline values. Pearson's correlation coefficient was computed to assess the relationship between severity of spasticity (preoperative baseline of total RMS value during passive stretch) and the effective stimulation parameters (frequency and stimulus strength). In all tests, a probability level of $P<0.05$ was considered statistically significant.

\section{Results}

\section{Effect of SCS on spasticity}

Overall, the benefit of our approach was dramatic. Based on our clinical rating scale used in the physiotherapeutic setting, six patients showed a marked and two patients a moderate response to long-term continuous stimulation.

Figure 1 summarizes the root mean square (RMS) amplitudes obtained with three passive stretch hip-knee reflexes in the presence of stimula- 
tion compared to preoperative baseline values. The total response of all muscles combined was statistically significant both in the left $(t=3.67$, $P=0.0040)$ and in the right $(t=3.76, P=0.0035)$ lower limbs. Even when broken down by muscles, all results were characterized by pronounced drops (Figure 1). The quadriceps muscle was the only parameter that failed to reach statistical significance both in the left $(P=0.1224)$ and in the right $(P=0.0951)$ leg. All other drops in RMS values were statistically significant (adductor: $P=0.0381$ / 0.0500, hamstring: $P=0.0047 / 0.0028$; anterior tibial: $P=0.0032 / 0.0037$; triceps surae: $P=0.0059 / 0.0005)$.

Similarly, Ashworth scores fell from a median baseline of $3.15(2.3-3.8)$ on the left and $3.2(2.5-$ $4.1)$ on the right down to $1.15(1.0-1.5 ; z=-2.5205$; $P=0.0117)$ and $1.3(1.0-1.6 ; \mathrm{z}=-2.5205, P=0.0117)$ respectively. This impressive decline in spasticity was also reflected in the pendulum test conducted in four

A

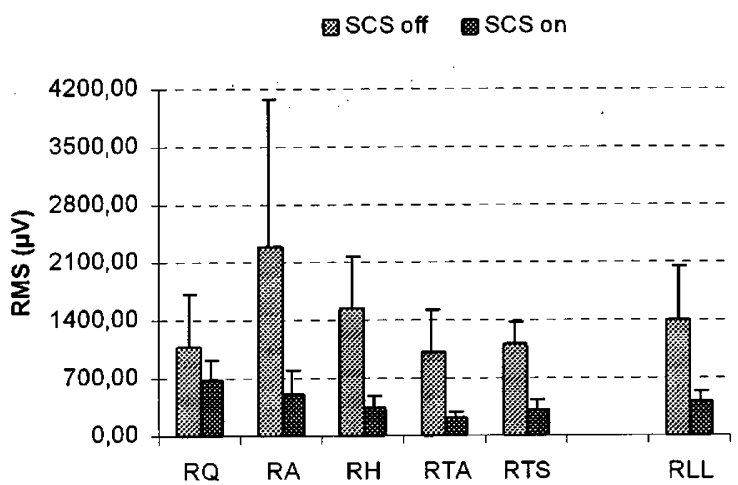

B

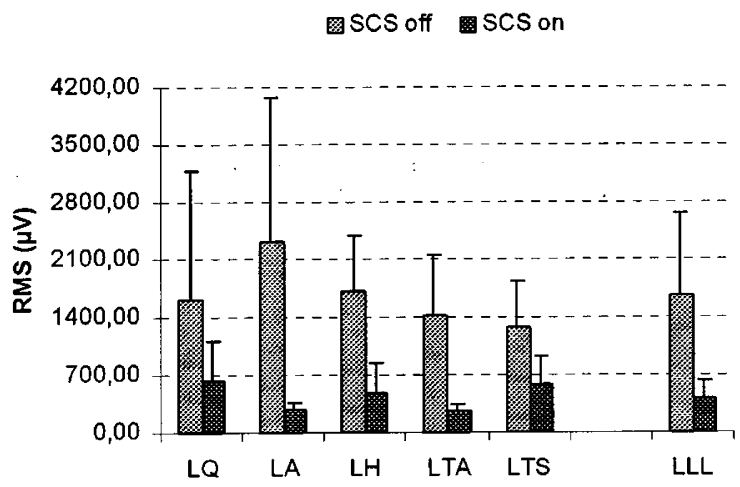

Figure 1 Mean RMS (root mean square) values and standard deviations obtained for three passive stretch hipknee reflexes in eight subjects. Results are broken down according to muscle types $(\mathrm{Q}=$ quadriceps, $\mathrm{A}=$ adductor, $\mathrm{H}=$ hamstring, $\mathrm{TA}=$ tibialis anterior, $\mathrm{TS}=$ triceps surae) in the lower right (A) and left (B) limbs. The bars on the right represent the total mean values for all muscles in lower left and right limbs (LLL, RLL) out of eight patients under conditions SCS-off (12 h or more) versus SCS-on (1 h or more). Figure 2 gives one illustrative example of a sharp drop in EMG activity associated with a fully restored swinging capacity of the right leg.

\section{Location and significance of the site of stimulation}

Table 2 summarizes the positions of the active cathode relative to the spine as determined by anteroposterior fluoroscopic visualization. Interestingly, all electrodes were found to be located between the bottom segment of TV 11 and the top segment of LV 1. What is more, the site of stimulation along the spinal cord, as determined by muscle twitches patterns, ${ }^{22}$ was such that the active cathode invariably stimulated the upper and middle portion of the lumbar cord. Two patients (\#4 and \#6) showed low-amplitude responses for tibial anterior and triceps surae in addition to high-

$\mathbf{A}$

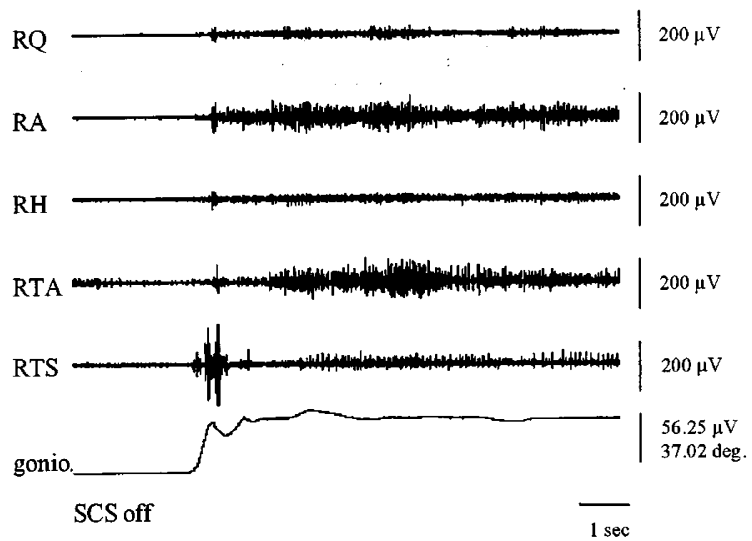

B

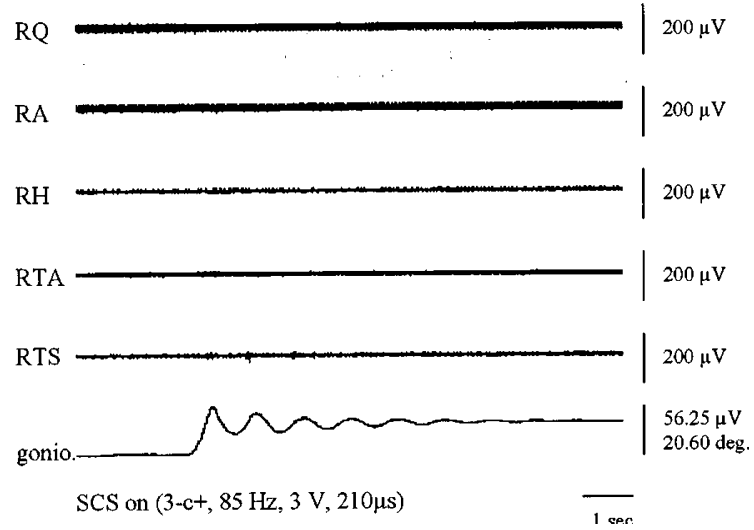

Figure 2 Pendulum test performed with the lower right limb of patient \#5. The upper chart (A) was obtained with the stimulator switched off, the lower chart (B) was obtained during active stimulation $(3-/ \mathrm{c}+, 85 \mathrm{~Hz}, 210 \mu \mathrm{s})$. Each chart also features a goniometer tracing of the knee movement: upward deflections indicate extension, downward deflections indicate flexion of the lower limb 
Table 2 Electrode positions relative to vertebrae and spinal cord segments

\begin{tabular}{|c|c|c|c|c|c|c|}
\hline \multirow[b]{2}{*}{ No } & \multicolumn{2}{|c|}{ Position of SCS electrode } & \multicolumn{4}{|c|}{$\begin{array}{c}\text { Parameter of Spinal Cord } \\
\text { Stimulation }\end{array}$} \\
\hline & Spine vertebra & Spinal cord & Polarity & Strength & Rate & Pulse width \\
\hline 1 & $\mathrm{TV} 12 / \mathrm{T}$ & ULS+MLS & $0-/ \mathrm{c}+$ & 3.5 & 50 & 210 \\
\hline 2 & $\mathrm{TV} 12 / \mathrm{B}$ & ULS+MLS & $2-10+$ & 3.0 & 70 & 210 \\
\hline 3 & TV $11 / \mathrm{B}$ & ULS+MLS & $2-/ c^{+}$ & 2.0 & 50 & 210 \\
\hline 4 & $\operatorname{LV} 1 / \mathrm{T}$ & ULS+MLS+LLS & $3-/ c+$ & 4.5 & 50 & 210 \\
\hline 5 & TV $12 / \mathrm{B}$ & ULS+MLS & $3-/ 0+$ & 2.7 & 90 & 210 \\
\hline 6 & TV $12 / M$ & ULS+MLS+LLS & $3-/ c^{+}$ & 3.5 & 75 & 210 \\
\hline 7 & $\mathrm{LV} 1 / \mathrm{T}$ & ULS+MLS & $2-/ c+$ & 6.0 & 100 & 210 \\
\hline 8 & $\mathrm{TV} 12 / \mathrm{T}^{*}$ & ULS+MLS & $0-/ 1-/ c^{+}$ & 7.1 & 90 & 210 \\
\hline
\end{tabular}

$\mathrm{TV}=$ thoracic vertebra; $\mathrm{LV}=$ lumbar vertebra; $\mathrm{B}=$ bottom; $\mathrm{T}=$ top; $\mathrm{T}^{*}=$ top plus one lead below at $\mathrm{M} ; \mathrm{ULS}=\mathrm{upper}$ lumbar spinal cord segment, MLS= middle lumbar spinal cord segment; LLS = lower lumbar spinal cord segment

amplitude responses for quadriceps, adductor and hamstring muscles.

The decisive role of correct placement becomes apparent when we use the same stimulation parameters on different stimulation sites. Figure 3 shows one representative result (patient \#5) obtained during passive stretch with a train of $50 \mathrm{~Hz}(210 \mathrm{~ms}, 3 \mathrm{~V})$ that was first applied to the upper border of TV 11 and then moved to the lower border of TV 12. In terms of spinal cord levels, these positions demonstrably corresponded to the lower thoracic and upper lumbar cord segments, respectively. It is apparent that the first position involved no improvement from baseline (Figure 3A) whereas in the second position spasticity was effectively controlled (Figure 3B).

Indeed we can generate marked differences in effect merely by switching contacts, ie without even moving the electrode itself. Figure 4 shows one representative example obtained in during passive stretch (patient \#8). In this case, we tested not only the different sites of stimulation (Figure 4A: $0-/ \mathrm{c}+$; Figure 4B: 3-/ $\mathrm{c}+)$ but additionally applied different frequencies $(50$, $80,100 \mathrm{~Hz})$ and stimulus strengths $(0,2,4,6,8,10 \mathrm{~V})$.

We observed that these variable parameters only made a difference when the correct site of stimulation was chosen. Stimulation with the most rostral lead (contact 0) as active cathode induced very similar RMS amplitudes both in the left and in the right leg regardless of stimulation parameters (Figure 4A). Contact 3 , by contrast, which is located $30 \mathrm{~mm}$ below contact 0 , not only had a significantly greater impact but also correlated closely with stimulation parameters, inducing significantly reduced RMS ampli-
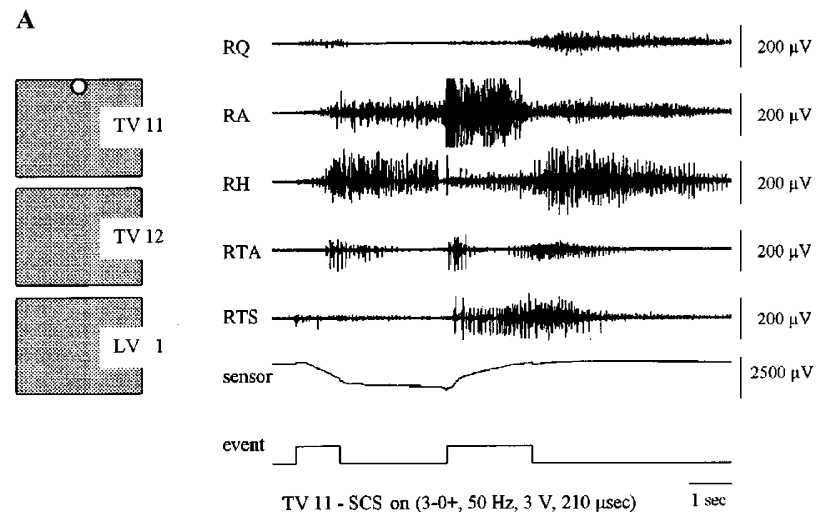

B
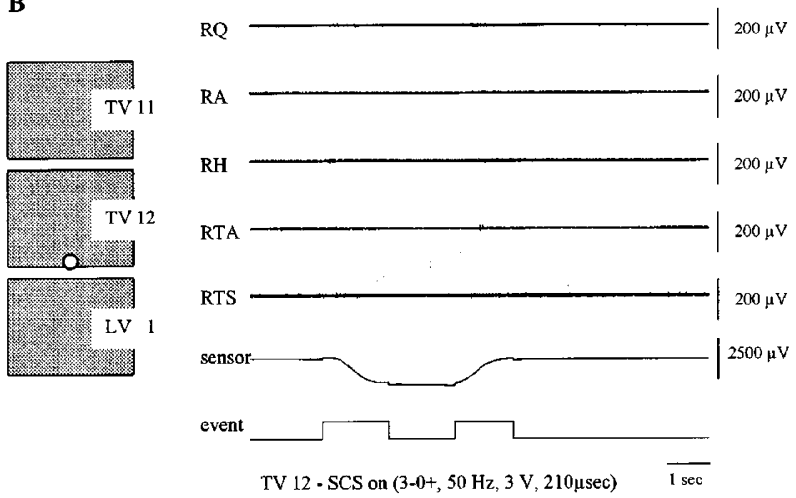

Figure 3 Differential effect of spinal cord stimulation with the cathode placed over two different sites. At the top of thoracic vertebra 11 there was no response (A) while hypertonia was completely eliminated at the bottom of thoracic vertebra 12 (B) 

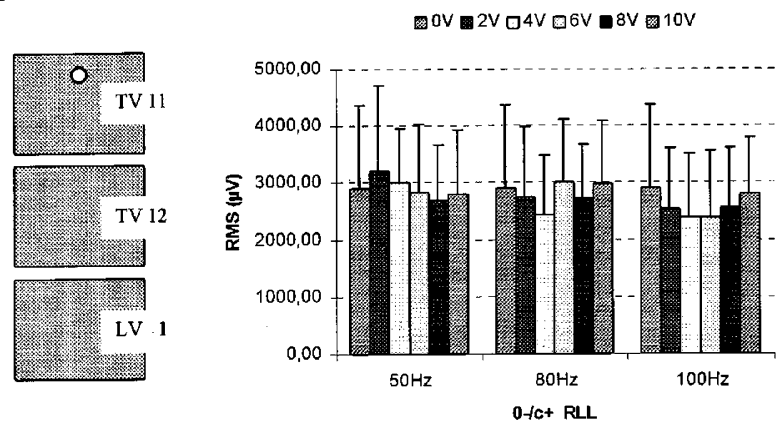

B
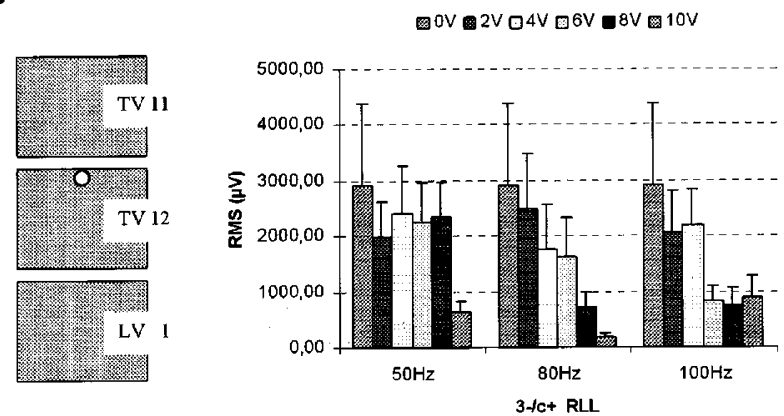

Figure 4 The bar charts on the right give an overview of mean RMS (root mean square) values and standard deviations obtained for two passive stretch hip-knee reflexes of the right lower limb (RLL) using different stimulation levels and frequencies. The distribution in the upper graph (A) was obtained with lead 0 as cathode, whereas in the lower graph (B) lead 3 was used as cathode. The rectangles on the left illustrate that lead 0 was placed over the upper portion of thoracic vertebra 11 and lead 3 over the top of thoracic vertebra 12

tudes at $50 \mathrm{~Hz} / 10 \mathrm{~V}(P=0.0118 / 0.0366)$, at $80 \mathrm{~Hz} / 8 \mathrm{~V}$ $(P=0.0116 / 0.0396)$ or at $100 \mathrm{~Hz} / 6 \mathrm{~V} \quad(P=0.0150 /$ 0.0456) (Figure 4B).

\section{Observations during long-term stimulation}

After continuous SCS had been carried on for a mean of $14.38( \pm 8.72)$ months, the mean stimulus strength was $4.04( \pm 1.37) \mathrm{V}$ and the mean frequency was 71.88 $( \pm 16.88) \mathrm{Hz}$. The pulse width of $210 \mu \mathrm{s}$ remained constant in all patients throughout the observation period, other parameters had to be adjusted in five cases (Table 2): the stimulation level was intensified in two and attenuated in another two patients after switching from bipolar to unipolar mode; the stimulation frequency was increased from 50 to $90 \mathrm{~Hz}$ in one patient after 4 months of continuous SCS; the polarity was changed in two patients after the electrode had migrated distally by one contact; and in one patient a second active cathode (contact 0 ) was added. At the end of the follow-up period, unipolar stimulation was used in six and bipolar stimulation in two patients. The patients were free to regulate the stimulus level in accordance with postural changes. The amplitudes given in Table 2 are mean values obtained with the patients in supine position.

Stimulation amplitudes and frequencies correlated, albeit not significantly, with the severity of spasms as reflected in total RMS values and Ashworth scores at study entry (ampl./freq. RMS left/right: $r=0.0645$ / 0.0262/0.4084/0.1292; ampl./freq. Ashworth left/right: $0.7065 / 0.5220 / 0.6917 / 0.6371)$. The more strongly affected patients required stronger stimuli and/or higher frequencies.

As we have indicated above, the polarity was switched in two patients after the electrode had migrated by one contact in distal direction. In one patient, the electrode was replaced three months postoperatively because of a broken lead. The pulse generator had to be replaced in three patients due to its limited life span, which mainly depends on the level of the generated stimulus. In one patient it was replaced twice.

Antispastic medication was discontinued in all patients but one as soon as continuous stimulation was in place. This patient (\#6) continues to be on baclofen, although the dose could be reduced from 125 to $50 \mathrm{mg}$, and tizanidine was discontinued altogether.

\section{Discussion}

Patients with spastic chronic SCI fall into two distinct groups characterized by either mild or severe spasticity. $^{25,26}$ Following reports that SCS applied below the level of the lesion is less effective in cases involving severe spasticity, ${ }^{10,11}$ we decided to explore the possibility of optimizing the approach by finding an appropriate site of stimulation. Encouraged by our previous finding of a central pattern generator (CPG) for locomotion in SCI patients, we hypothesized that there may exist a window for activating the lumbar spinal network at the level of the second dorsal lumbar roots. ${ }^{16}$ This network can generate excitation and inhibition not only in turns but also one without the other. We therefore expected that by focusing the stimulus on the upper lumbar cord segment we should eventually succeed in controlling even severe spasticity of the lower limb.

The antispastic effect of SCS described in this communication was apparent from both the clinical and the experimental setup. Our clinical rating scale was applied by physiotherapists in an independent setting and revealed a marked effect of SCS in six out of eight patients, while the remaining two patients still showed a moderate effect. Although these differences were not reflected in our EMG data, which invariably showed significant improvements in all patients, we presume that this slight discrepancy is within the normal range of subjective observation deviating from objective measurement. ${ }^{26-28}$

Even the EMG activities separately obtained for the various muscle groups were consistently characterized 
by significant improvements. Only the improvements recorded for both quadriceps muscles failed to reach statistical significance. One might assume that this difference is due to a shorter stretch compared to hamstring muscles, but then the adductor muscle group has an even shorter stretch than quadriceps during passive flexion of the knee. It therefore appears more plausible to attribute this difference between quadriceps and adductors to a central mechanism.

Passive movements are characterized by the absence of reciprocal innervation while antagonistic muscle groups are coactivated to the point of completely suppressing the activity of motor units (Figure 3A). Severe spasticity in SCI has been shown to involve a dominant pattern of diffuse, plurisegmental, increased motor units output of the spinal cord. ${ }^{29}$ Reciprocal inhibition is only present in the early phase of tendon jerk response, manifesting itself as a silent interval in the antagonistic muscle group. ${ }^{30}$ In patients with severe spasticity, it is followed by a prolonged motor units 'afterdischarge' induced by phasic tonic stretch reflex or cutaneomuscular reflex responses that will radiate to ipsilateral and contralateral limbs. This phenomenon of motor units activity within spastic muscles being widely distributed is caused by an increased excitatory activity of the plurisegmental interneuron network, whose role is to spread excitation within the spinal cord below the level of the injury. ${ }^{31,32}$

In accordance with these observations, our study revealed that SCS induced a generalized (diffuse) suppression, which suggests that the adopted approach of spinal cord stimulation enhanced the activity of an inhibitory plurisegmental network situated within the lumbar cord. ${ }^{33}$

It has been shown repeatedly that mild forms of spasticity can be effectively controlled by placing the electrode below the level of the spinal cord lesion. ${ }^{2,5,9,10}$ Why this approach has failed in cases of severe spasticity becomes clear when we realize how heavily the antispastic effect of SCS depends on the site of stimulation (Figures 3 and 4). Rather than just placing the electrode below the level of the lesion, it is essential that the stimulation site targets the dorsal roots of the upper lumbar cord segment. As a possible explanation for this difference, we suggest that the previously adopted approach can only activate non-specific inhibitory mechanisms within the dorsal column-brainstem-spinal loop $^{34}$ whereas our own approach succeeds in activating a specific inhibitory mechanism within the lumbar spinal cord. Because the remarkable antispastic effect of targeting the dorsal roots of the upper lumbar cord segment was absent when the stimulus was applied to the neighboring lower thoracic or the lower lumbar segment, we suggest that there exists a network within the lumbar cord that can be activated by exciting the posterior structures of the L2 segment. The same specific dependence on the site of stimulation is present to elicit activity of a spinal pattern generator for locomotion in chronic paraplegic patients. ${ }^{16}$

Thus there apparently exist two different sites of stimulation, one of which is suitable only for SCI patients with mild spasticity and incomplete lesions. In this type of situation, stimulation below the level of the lesion may be a perfectly serviceable approach, but in situations of severe spasticity in the lower limbs it is essential to selectively stimulate upper lumbar dorsal roots. A detailed description of how the correct site of upper lumbar stimulation can be located is given in the first paper of this series. ${ }^{22}$

All other stimulation parameters are variable. Frequencies and amplitudes must be adapted on a case-by-case basis, regardless of whether the electrode is operated in unipolar or bipolar mode. We did, however, observe a general trend that the more severely affected patients required either higher amplitudes or higher frequencies.

Looking to the future, there is a need to develop new epidural electrodes specifically designed for the stimulation of lumbar cord dorsal roots. This design would involve an extended reach to cover multiple sites not in the vertical but in the horizontal plane. The results of this study bear testimony to the fact that we are gradually learning how to restore normal functions of the spinal cord by identifying specific sites of stimulation.

\section{Acknowledgements}

We wish to thank all patients who participated in the study and the physicians who referred them to our program. We are indebted to Ms Alesch, Ms Auer, Ms Preinfalk and Dr Lamp for their help and technical support. This work was supported by the Ludwig Boltzmann Institute for Restorative Neurology and Neuromodulation in Vienna, Austria, and by a research grant of the Kent Waldrep National Paralysis Foundation in Dallas, Texas, USA.

\section{References}

1 Cook AW, Weinstein SP. Chronic dorsal column stimulation for the treatment of movement disorders. NY State J Med 1973; 73: $2868-2872$.

2 Illis LS. Spinal cord stimulation in spasticity and bladder dysfunction. In: Illis LS (ed). Spinal Cord Dysfunction. Vol 3. Oxford University Press: Oxford, New York, Tokyo, 1992; pp $294-303$

3 Dimitrijevic MR, Faganel J. Spinal cord stimulation for the treatment of movement disorders. In: Lazorthes Y, Upton ARM (eds). Neurostimulation: An Overview. Futura: Mt. Kisco, NY, 1985 , pp $147-172$.

4 Gybels J, Van Roost D. Spinal cord stimulation for spasticity. In: Sindou M, Abbott R, Keravel Y (eds). Neurosurgery for Spasticity. Springer-Verlag: Wien, 1991, pp 73-81.

5 Gybels J, Van Roost D. Spinal cord stimulation for the modification of dystonic and hyperkinetic conditions: A critical review. In: Eccles JC, Dimitrijevic MR (eds). Recent Achievements in Restorative Neurology. Karger: Basel, 1985, pp 56-70. 
6 Cioni B, Meglio M, Zamponi A. The effect of spinal cord stimulation on motor performance in hemiplegics. Stereotact Funct Neurosurg 1989; 52: $142-152$.

7 Richardson RR, McLone DG. Percutaneous epidural neurostimulation for paraplegic spasticity. Surg Neurol 1978; 9: 153 - 155.

8 Siegfried J, Lazorthes Y, Broggi G. Electrical spinal cord stimulation for spastic movement disorders. Proc. 3rd Int. Meeting on Spinal Cord Stimulation, Houston. Appl Neurophysiol 1980; 44: $77-92$.

9 Dimitrijevic MM et al. Spinal cord stimulation for the control of spasticity in patients with chronic spinal cord injury: I. Clinical observations. Cent Nerv Syst Trauma 1986; 3: $129-144$.

10 Barolat $\mathrm{G}$ et al. Epidural spinal cord stimulation in the management of spasms in spinal cord injury. A prospective study. Stereotact Funct Neurosurg 1995; 64: 153-164.

11 Dimitrejevic MR et al. Spinal cord stimulation for the control of spasticity in patients with chronic spinal cord injury: II. Neurophysiological observations. Cent Nerv Syst Trauma 1986; 3: $145-152$.

12 Barolat G, Ketcik B, He J. Long-term outcome of spinal cord stimulation for chronic pain management. Neuromodulation 1998; 1: $19-29$.

13 Dimitrijevic MR. Residual motor functions in spinal cord injury. In: Waxman SG (ed). Advances in Neurology Vol. 47: Functional Recovery in Neurological Disease. Raven Press: New York, 1988, pp $139-155$.

14 Dimitrijevic MR, McKay WB, Sherwood AM. Motor control physiology below spinal cord injury: residual volitional control of motor units in paretic and paralyzed muscles. In: FJ Seil (ed). Neuronal Regeneration, Reorganization and Repair, Raven Press: New York, 1997, pp 335-345.

15 Dimitrijevic MR. Motor control in human spinal cord injury. In: Stalberg E, Sharma HS, Olsson Y (eds). Spinal Cord Monitoring. Springer: Wien, New York, 1998, pp 409-420.

16 Dimitrijevic MR, Gerasimenko Y, Pinter MM. Evidence for a spinal central pattern generator in humans. In: Kiehn O, HarrisWarrick RM, Jordan LM, Hultborn H, Kudo N (eds). Ann NY Acad Sci Vol. 860, Neuronal Mechanism for Generating Locomotor Activity 1998, pp 360-376.

17 Lee KC, Carson L, Kinnin E, Patterson V. The Ashworth Scale: A reliable and reproducible method of measuring spasticity. $J$ Neuro Rehab 1989; 3: 205-209.

18 Ditunno JF, Young W, Donovan WH, Creasey G. The international standard booklet for neurological and functional classification of spinal cord injury. Paraplegia 1994; 32: 70-80.

19 Dimitrijevic MR, Hsu CY, McKay WB. Neurophysiological assessment of spinal cord and head injury. J Neurotrauma 1992; 9 (Suppl 1): $293-300$.
20 Sherwood AM, McKay B, Dimitrijevic MR. Motor control after spinal cord injury: assessment using surface EMG. Muscle Nerve 1996; 19: $966-979$.

21 Dimitrijevic MR, Faganel J, Sharkey PC, Sherwood AM. Study of sensation and muscle twitch responses to spinal cord stimulation. Int Rehab Med 1980; 2: 76-81.

22 Murg M, Binder H, Dimitrijevic MR. Epidural electric stimulation of posterior structures of the human lumbar spinal cord: 1. Muscle twitches - a functional method to define the site of stimulation. Spinal Cord 2000; (in press).

23 Campos RJ, Dimitrijevic MM, Faganel J, Sharkey PC. Clinical evaluation of the effect of spinal cord stimulation on motor performance in patients with upper motor neuron lesion. Appl Neurophysiol 1981; 44: 141 - 151

24 Vodovnik L, Bowman BR, Bajd T. Dynamics of spastic knee joint. Med Biol Eng Comput 1984; 22: 63-69.

25 Franzoi AC, Castro C, Cardone C. Isokinetic assessment of spasticity in subjects with traumatic spinal cord injury (ASIA A). Spinal Cord 1999; 37: 416-420.

26 Akman MN et al. Assessment of spasticity using isokinetic dynamometry in patients with spinal cord injury. Spinal Cord 1999; 37: 638-643.

27 Bohannon RW, Smith MB. Interrater reliability of a modified Ashworth scale of muscle spasticity. Phys Ther 1987; 67: $206-$ 207

28 Haas BM, Berström E, Jamous A, Bennie A. The interrater reliability of the original and of the modified Ashworth scale for assessment of spasticity in patients with spinal cord injury. Spinal Cord 1996; 34: 560-564.

29 Dimitrijevic MR, Nathan PW. Studies of spasticity in man. 1 Some features of spasticity. Brain 1967; 90: $1-30$.

30 Dimitrijevic MR, Nathan PW. Studies of spasticity in man. 2. Analysis of stretch reflexes in spasticity. Brain 1967; 90: 333 - 358

31 Dimitrijevic MR, Faganel J, Lehmkuhl D, Sherwood A. Motor control in man after partial or complete spinal cord injury. In: Desmedt JE (ed). Motor Control Mechanisms in Health and Disease. Raven Press: New York, 1983, pp 915-926.

32 Dimitrijevic MR. Evaluation and treatment of spasticity. J Neuro Rehab 1995; 9: $97-110$.

33 Dimitrijevic MR et al. Habituation: Effects of regular and stochastic stimulation. J Neurol Neurosurg Psychiat 1972; 35: $234-242$

34 Saade NE, Tabet MS, Atweh SF, Jabbur SJ. Modulation of segmental mechanisms by activation of a dorsal column brainstem spinal loop. Brain Res 1984; 310: 180 - 184. 